\title{
Higher-Order Overtone Thickness-Shear Vibrations of Multilayered Thin-Film Acoustic Wave Resonators and Angular Rate Sensing
}

\author{
Hui Chen, Ji Wang and Jianke Du \\ Piezoelectric Device Laboratory, School of Mechanical Engineering and Mechanics, Ningbo University, Ningbo, \\ Zhejiang 315211, China.
}

\begin{abstract}
Jiashi Yang
Piezoelectric Device Laboratory, School of Mechanical Engineering and Mechanics, Ningbo University, Ningbo, Zhejiang 315211, China.

Department of Mechanical and Materials Engineering, University of Nebraska-Lincoln, Lincoln, NE 68588-0526, USA.
\end{abstract}

(Received 6 September 2015; accepted 26 April 2017)

\begin{abstract}
We propose a new structure for piezoelectric gyroscopes. It is made from multilayered thin films of $\mathrm{AlN}$ or $\mathrm{ZnO}$ with alternating $c$-axes along the film thickness. It is shown theoretically that when such a film is electrically driven into higher-order overtone thickness-shear vibration in one of the two in-plane directions of the film and is rotating about the film normal, the Coriolis force due to the rotation causes a higher-order overtone thicknessshear vibration in a perpendicular direction with an electrical output that can be used to measure the angular rate of the rotation. Different from existing thickness-shear mode piezoelectric gyroscopes which are based on the fundamental or the second overtone thickness-shear mode, the proposed gyroscope operates with higher-order overtone thickness-shear modes with higher frequencies and hence potentially higher sensitivity. Because of the overtone modes, the Coriolis force acting on the gyroscope forms a self-equilibrated system and does not transmit a net force or torque to the mounting structure. This implies higher device quality factor and better performance.
\end{abstract}

\section{INTRODUCTION}

Piezoelectric materials can be used to make vibratory gyroscopes or angular rate sensors. ${ }^{1}$ They make use of two vibration modes of a piezoelectric body, in which material particles moving in perpendicular directions are coupled by the Coriolis force when the gyroscope is rotating. When the gyroscope is excited into vibration by an applied alternating voltage in one of the two modes (the primary mode) and rotates with an angular rate, the Coriolis force from the primary mode excites the other mode (the secondary mode) through which the angular rate can be detected from electrical signals (voltage or current) accompanying the secondary motion. Many piezoelectric gyroscopes are based on flexural vibrations in perpendicular directions of beams. References on beam gyroscopes can be found in a review article. ${ }^{2}$

This paper is concerned with piezoelectric gyroscopes operating with thickness-shear (TSh) modes in perpendicular directions of a plate ${ }^{3-9}$ which have several advantages. TSh modes of a plate have higher frequencies than flexural modes of a beam with a comparable size. For vibratory gyroscopes, higher frequencies imply potentially higher sensitivity. ${ }^{2}$ In addition, TSh modes have an important behaviour called energy trapping, ${ }^{4,5}$ in which the vibration is confined in the central part of the plate. Near the edges of the plate there is little vibration where mounting can be designed without affecting the operation of the device. Most TSh mode gyroscopes operate with the fundamental TSh mode of a plate. ${ }^{3-8}$ Recently, it was pointed out ${ }^{9}$ that for the fundamental TSh mode, although the energy trapping confines the TSh vibration to the central part of the plate, the Coriolis force due to rotation forms a torque on the gyroscope which has to be balanced by a reactive torque from the mounting or support. This causes energy leakage to the support and lowers the device quality factor. A TSh piezoelectric gyroscope was then proposed ${ }^{9}$ based on the second overtone TSh mode of a lithium niobate plate with an inversion layer whose Coriolis force forms a self-equilibrated system and does not transmit to the support, which is helpful for raising the device quality factor.

The lithium niobate plate with an inversion layer ${ }^{9}$ was effective for exciting the second overtone of TSh, but not the higher-order TSh overtones. The higher-order TSh overtones are also attractive for gyroscope applications. They have higher frequencies and hence potentially higher sensitivity. ${ }^{2}$ Recently, micromachined, multilayered thin films of AlN and $\mathrm{ZnO}$ have been produced for acoustic wave resonator applications. ${ }^{10}$ They belong to micromachined devices ${ }^{11-15}$ and are much thinner than conventional crystal plate TSh gyroscopes of quartz or lithium niobate. This leads to higher frequencies and potentially higher sensitivity. In these multilayered thin films, the $c$-axis of each layer can assume different and independent directions. This offers the opportunity for exciting higher-order overtone TSh modes ${ }^{16}$ conveniently with even higher frequencies and self-equilibrated Coriolis forces. In this paper we demonstrate theoretically the advantages of using a multilayered thin film of $\mathrm{AlN}$ or $\mathrm{ZnO}$ to make a piezoelectric gyroscope operating with higher-order overtone TSh modes.

\section{STRUCTURE}

Consider the four-layered thin film of $\mathrm{AlN}$ or $\mathrm{ZnO}$ in Fig. 1 as an example. The reference frame is attached to the film and is rotating with it. The analysis below can be generalized to thin films with more layers. The thicknesses of the 


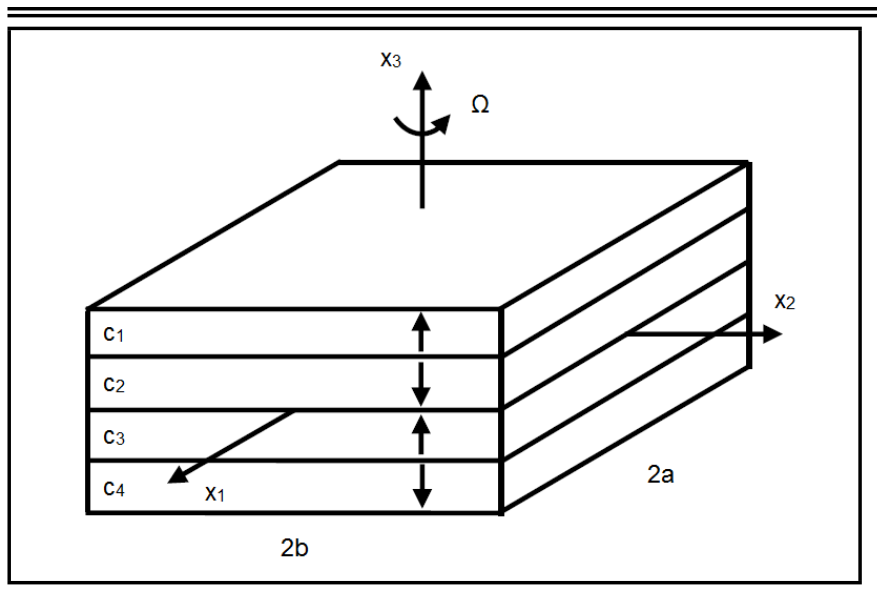

Figure 1. A four-layered thin film of $\mathrm{AlN}$ or $\mathrm{ZnO}$ with alternating $c$-axes.

layers are shown in $c_{1}$ through $c_{4}$, and the $c$-axes of the layers are either along $x_{3}$ or minus $x_{3}$, as shown by the arrows in the figure. The film has four separate electrodes on its four sides. Across the electrodes where $x_{1}= \pm a$, a time-harmonic driving voltage, $V_{1}$, is applied, producing an in-plane or lateral driving electric field $E_{1}=-V_{1} /(2 a)$.

Due to the specific material anisotropy of AlN or $\mathrm{ZnO}$ which both belong to the crystal class of $6 \mathrm{~mm}, E_{1}$ drives the plate into the primary TSh motion described by $u_{1}\left(x_{3}, t\right)$. When the plate is rotating about the $x_{3}$-axis with an unknown angular rate $\Omega$ which is to be measured, the related Coriolis force drives the plate into the secondary TSh motion described by $u_{2}\left(x_{3}, t\right)$ which produces an electric field $E_{2}$ and a related output voltage $V_{2}$ across the electrodes at $x_{2}= \pm b$, with $E_{2}=-V_{2} /(2 b)$. The electrodes at $x_{2}= \pm b$ are joined by an output circuit whose impedance is $Z$ in harmonic motions. The output current in this circuit is denoted by $I$. It will be shown that $V_{2}$ (and $I$ ) are related to $\Omega$ and can be used to measure it.

\section{GOVERNING EQUATIONS}

Plate TSh devices have large length/thickness and width/thickness ratios in order to produce nearly pure TSh modes with fewer couplings to other unwanted modes and fewer in-plane variations along $x_{1}$ and $x_{2}$. Therefore, we neglect edge effects and use a one-dimensional model depending on the film thickness coordinate, $x_{3}$, and time. This is sufficient for showing the operation principle and the basic behaviour of the proposed gyroscope. Since the material changes from layer to layer, we need to treat each layer separately and then apply continuity conditions at their interfaces and boundary conditions at the film's top and bottom surfaces.

For TSh vibrations of the top layer with the $c$-axis along $x_{3}$, the relevant displacement and electric potential fields are: ${ }^{9}$

$$
\begin{array}{r}
u_{1}=u_{1}\left(x_{3}\right) e^{i \omega t} ; \\
u_{2}=u_{2}\left(x_{3}\right) e^{i \omega t} ; \\
u_{3}=0 ; \\
\phi=-E_{1} x_{1} e^{i \omega t}-E_{2} x_{2} e^{i \omega t} .
\end{array}
$$

The relevant equations of motion for a rotating piezoelectric body take the following form: 2,9

$$
T_{31,3}=\rho\left(\frac{\partial^{2} u_{1}}{\partial t^{2}}-2 \Omega \frac{\partial u_{2}}{\partial t}-\Omega^{2} u_{1}\right) ;
$$

$$
T_{32,3}=\rho\left(\frac{\partial^{2} u_{2}}{\partial t^{2}}+2 \Omega \frac{\partial u_{1}}{\partial t}-\Omega^{2} u_{2}\right) ;
$$

where the shear stresses $T_{31}$ and $T_{32}$ are related to the displacement gradients and the electric fields through: ${ }^{9,17}$

$$
\begin{aligned}
& T_{31}=c_{55} u_{1,3}-e_{15} E_{1} \\
& T_{32}=c_{55} u_{2,3}-e_{15} E_{2} .
\end{aligned}
$$

Since for steady-state motions all of the electromechanical fields are time harmonic, we use the usual complex notation. The same exponential time factor is dropped for simplicity. To calculate the charge on the driving and output electrodes, we need: ${ }^{9,17}$

$$
\begin{aligned}
& D_{1}=e_{15} u_{1,3}+\varepsilon_{11} E_{1} ; \\
& D_{2}=e_{15} u_{2,3}+\varepsilon_{11} E_{2} .
\end{aligned}
$$

The substitution of Eq. (3) into Eq. (2) gives:

$$
\begin{aligned}
& c_{55} u_{1,33}=\rho\left(-\omega^{2} u_{1}-2 i \omega \Omega u_{2}-\Omega^{2} u_{1}\right) \\
& c_{55} u_{2,33}=\rho\left(-\omega^{2} u_{2}+2 i \omega \Omega u_{1}-\Omega^{2} u_{2}\right) .
\end{aligned}
$$

The above equations are also valid for the third layer from the top with the $c$-axis also along $x_{3}$.

Similarly, for the second layer from the top with the $c$-axis along minus $x_{3}$, Eqs. (1), (2) and (5) remain the same. Since the piezoelectric constants change their signs when the $c$-axis is reversed, Eqs. (3) and (4) become:

$$
\begin{aligned}
& T_{31}=c_{55} u_{1,3}+e_{15} E_{1} ; \\
& T_{32}=c_{55} u_{2,3}+e_{15} E_{2} ;
\end{aligned}
$$

and:

$$
\begin{aligned}
& D_{1}=-e_{15} u_{1,3}+\varepsilon_{11} E_{1} ; \\
& D_{2}=-e_{15} u_{2,3}+\varepsilon_{11} E_{2} .
\end{aligned}
$$

The equations for the bottom layer are the same as those of the second layer from the top.

The traction-free boundary conditions at the film top surface are:

$$
\begin{aligned}
& T_{31}\left(c_{1}+c_{2}\right)=0 ; \\
& T_{32}\left(c_{1}+c_{2}\right)=0 .
\end{aligned}
$$

The continuity conditions at the interfaces among the four layers with $x_{3}=c_{2}, x_{3}=0$, or $x_{3}=-c_{3}$ are:

$$
\begin{array}{r}
u_{1}\left(c_{2}^{+}\right)=u_{1}\left(c_{2}^{-}\right) ; \quad u_{2}\left(c_{2}^{+}\right)=u_{2}\left(c_{2}^{-}\right) ; \\
T_{31}\left(c_{2}^{+}\right)=T_{31}\left(c_{2}^{-}\right) ; \quad T_{32}\left(c_{2}^{+}\right)=T_{32}\left(c_{2}^{-}\right) ; \\
u_{1}\left(0^{+}\right)=u_{1}\left(0^{-}\right) ; \quad u_{2}\left(0^{+}\right)=u_{2}\left(0^{-}\right) ; \\
T_{31}\left(0^{+}\right)=T_{31}\left(0^{-}\right) ; \quad T_{32}\left(0^{+}\right)=T_{32}\left(0^{-}\right) ; \\
u_{1}\left(-c_{3}^{+}\right)=u_{1}\left(-c_{3}^{-}\right) ; \quad u_{2}\left(-c_{3}^{+}\right)=u_{2}\left(-c_{3}^{-}\right) ; \\
T_{31}\left(-c_{3}^{+}\right)=T_{31}\left(-c_{3}^{-}\right) ; \quad T_{32}\left(-c_{3}^{+}\right)=T_{32}\left(-c_{3}^{-}\right) .
\end{array}
$$


The boundary conditions at the film bottom surface are:

$$
\begin{aligned}
& T_{31}\left(-c_{3}-c_{4}\right)=0 \\
& T_{32}\left(-c_{3}-c_{4}\right)=0 .
\end{aligned}
$$
by:

The free charge on the output electrode at $x_{2}=b$ is given

$$
q=2 a \int_{-c_{3}-c_{4}}^{c_{1}+c_{2}}-D_{2} \partial x_{3} .
$$

Then the current that flows out of the electrode is:

$$
I=-\frac{\partial q}{\partial t}=-i \omega q
$$

The equation for the output circuit is:

$$
V_{2}=I Z \text {. }
$$

There are sixteen boundary and continuity conditions in Eqs. (8), (9), and (10), plus the circuit equation in Eq. (13). They will be used to determine the sixteen undetermined constants in the solutions from Eq. (5) (four from each layer) in the next section, plus the unknown output voltage $V_{2}$.

\section{ANALYTICAL SOLUTION}

Consider the top layer first. Equation (5) is a system of ordinary differential equations with constant coefficients. Its general solution can be obtained in a systematic manner without any mathematical challenge. The result can be written as:

$$
\begin{array}{r}
u_{1}=\sum_{m=1}^{4} A^{(m)} e^{\left(\zeta^{(m)} x_{3}\right)} ; \\
u_{2}=\sum_{m=1}^{4} \alpha^{(m)} A^{(m)} e^{\left(\zeta^{(m)} x_{3}\right)} ;
\end{array}
$$

where $A^{(m)}$ ( $m=1,2,3$ and 4 ) are four undetermined constants. The values $\alpha^{(m)}$ are determined from Eq. (5) as:

$$
\alpha^{(m)}=\frac{2 i \rho \omega \Omega}{c_{55}\left(\zeta^{(m)}\right)^{2}+\rho \omega^{2}+\rho \Omega^{2}} .
$$

$\zeta^{(m)}$ are the four roots of:

$$
\left|\begin{array}{cc}
c_{55} \zeta^{2}+\rho \omega^{2}+\rho \Omega^{2} & 2 i \rho \omega \Omega \\
-2 i \rho \omega \Omega & c_{55} \zeta^{2}+\rho \omega^{2}+\rho \Omega^{2}
\end{array}\right|=0 .
$$

Similarly, for the second layer from the top, the general solution to Eq. (5) can be written as:

$$
\begin{array}{r}
u_{1}=\sum_{m=1}^{4} \bar{A}^{(m)} e^{\left(\zeta^{(m)} x_{3}\right)} ; \\
u_{2}=\sum_{m=1}^{4} \alpha^{(m)} \bar{A}^{(m)} e^{\left(\zeta^{(m)} x_{3}\right)} ;
\end{array}
$$

where $\bar{A}^{(m)}$ are four undetermined constants. For the third layer from the top, the general solution to Eq. (5) can be written as:

$$
\begin{array}{r}
u_{1}=\sum_{m=1}^{4} \hat{A}^{(m)} e^{\left(\zeta^{(m)} x_{3}\right)} ; \\
u_{2}=\sum_{m=1}^{4} \alpha^{(m)} \hat{A}^{(m)} e^{\left(\zeta^{(m)} x_{3}\right)} ;
\end{array}
$$

where $\hat{A}^{(m)}$ are four undetermined constants. For the fourth layer from the top, the general solution to Eq. (5) can be written as:

$$
\begin{array}{r}
u_{1}=\sum_{m=1}^{4} \tilde{A}^{(m)} e^{\left(\zeta^{(m)} x_{3}\right)} ; \\
u_{2}=\sum_{m=1}^{4} \alpha^{(m)} \tilde{A}^{(m)} e^{\left(\zeta^{(m)} x_{3}\right)} ;
\end{array}
$$

where $\tilde{A}^{(m)}$ are four undetermined constants.

With Eqs. (14) and (17) through (19), the output current $I$ can be calculated from Eq. (12) using Eqs. (4), (7) and (11). The substitution of Eqs. (14), (17) through (19) and the expression of the current $I$ into Eqs. (8), (9), and (10) and Eq. (13) results in seventeen equations for $A^{(m)}, \bar{A}^{(m)}, \hat{A}^{(m)}, \tilde{A}^{(m)}$ and $V_{2}$. These equations are solved on a computer.

\section{NUMERICAL RESULTS}

As a numerical example, consider an AlN film with $c_{1}=$ $c_{2}=c_{3}=c_{4}=0.01 \mathrm{~mm}$ and $2 a=2 b=1 \mathrm{~mm}$. The length/thickness ratio of the film is 25 , indicating a very thin film, hence edge effects can be neglected. The elastic, piezoelectric, and dielectric constants of AlN are available. ${ }^{18}$ To take material damping into consideration, in the numerical calculation, a complex elastic stiffness $c_{55}\left(1+i Q^{-1}\right)$ was introduced where $i$ is the imaginary unit and $Q$ is a real, positive, and large number (the material quality factor). $Q=10^{2}$ was used. We also introduced the following impedance $Z_{0}$ and frequency $\omega_{0}$ as units for the load impedance $Z$ and the driving frequency $\omega$ :

$$
\begin{array}{r}
Z_{0}=\frac{1}{i \omega_{0} C_{0}} ; \\
C_{0}=\frac{\varepsilon_{11}\left(c_{1}+c_{2}+c_{3}+c_{4}\right) 2 b}{2 a} ; \\
\omega_{0}=\frac{\pi}{c_{1}+c_{2}+c_{3}+c_{4}} \sqrt{\frac{c_{55}}{\rho}} ;
\end{array}
$$

where $\omega_{0}$ is very close to the fundamental TSh frequency of the four-layered film. In Eq.(20), $c_{55}$ is kept real. With the above data, we found that $\omega_{0}=4.7252 \times 10^{8} 1 / \mathrm{s}$ and $Z_{0}=$ $-7.4694 \times 10^{5} i \mathrm{ohm}$, and $\omega_{0}$ is an order of magnitude higher than that of a typical quartz or lithium niobate plate TSh mode resonator. For the applied voltage, $V_{1}=1$ volt was used in the calculation.

Figure 2 shows the normalized output voltage versus the normalized driving frequency. At some frequencies there are significant output (the peaks in the figure), indicating resonances. The gyroscope is supposed to operate with the fourth overtone TSh mode because the specific film under consideration has four layers with a special arrangement of the orientations of the $c$-axes. Therefore, we expect the first peak, or 


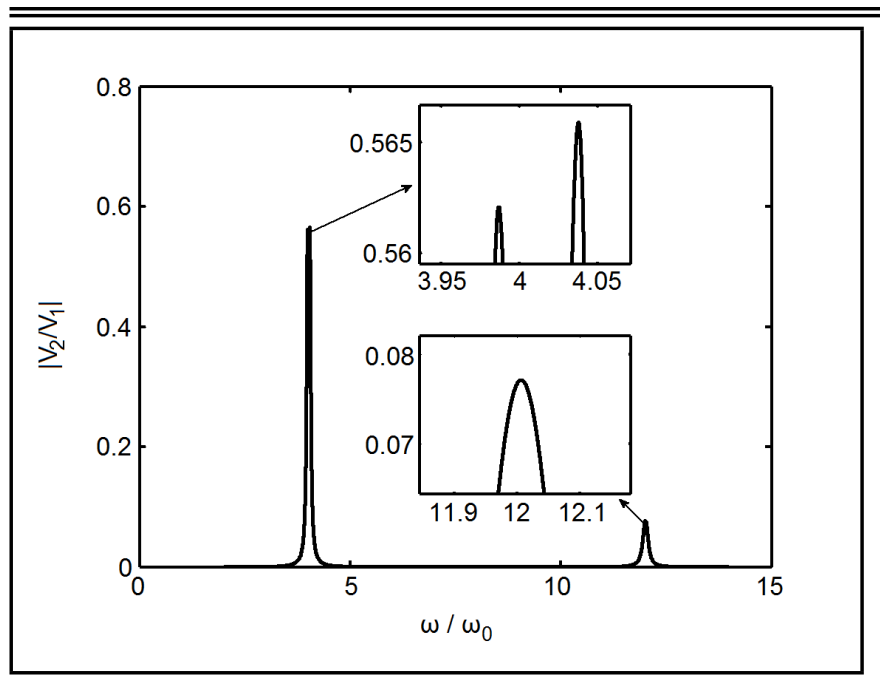

Figure 2. Output voltage $V_{2}$ versus driving frequency $\omega\left(\Omega=0.02 \omega_{0}, Z=\right.$ $\left.0.3 Z_{0}\right)$.

resonance, to be close to 4 which is indeed shown in the figure. A closer look at the peak near $\frac{\omega}{\omega_{0}}=4$ shows that in fact it consists of two peaks when magnified locally (also shown in the figure). Despite that the peak near $\frac{\omega}{\omega_{0}}=12$ still appears as one peak when magnified, we believe that it in fact consists of two overlapped peaks from our knowledge on gyroscopes in general. $^{2}$

Next, we examine the displacement distribution along the film thickness corresponding to the peaks in Fig. 2. Figure 3 (a) and (b) show $u_{1}\left(x_{3}\right)$ corresponding to the left peak and the right peak near $\frac{\omega}{\omega_{0}}=4$, respectively. $u_{2}\left(x_{3}\right)$ is qualitatively similar. When the film is not rotating, $u_{1}$ and $u_{2}$ represent two uncoupled higher-order overtone TSh modes along $x_{1}$ and $x_{2}$, respectively. One has $u_{1}$ only and the other has $u_{2}$ only. When the film is rotating about its normal, $u_{1}$ and $u_{2}$ are coupled by the Coriolis force. In Fig. 3 (a) the imaginary part of $u_{1}$ is much larger than the real part, but in (b) the situation is the opposite. This indicates a phase difference between the two modes in (a) and (b). The distribution of the velocity $\partial u_{1} / \partial t=i \omega u_{1}$ along the film thickness is similar to that of $u_{1}$. In Fig. 3 , the area bounded by $u_{1}$ with $u_{1}>0$ is the same as the area with $u_{1}<0$. In addition, $u_{1}$ is symmetric about the film middle plane. Therefore, we have:

$$
\begin{aligned}
\int_{-c_{3}-c_{4}}^{c_{1}+c_{2}} u_{1} \partial x_{3} & =0 \\
\int_{-c_{3}-c_{4}}^{c_{1}+c_{2}} x_{3} u_{1} \partial x_{3} & =0 .
\end{aligned}
$$

Since the Coriolis force associated with $\partial u_{1} / \partial t$ is proportional to $\partial u_{1} / \partial t$ and hence proportional to $u_{1}$, Eq. (21) implies that the resultant and the moment of the Coriolis force associated with $u_{1}$ are both zero. Therefore, the Coriolis force forms a self-equilibrated system and does not require a reactive force or moment from the supports of the device. We note that the Coriolis force is in fact not a real force. It is a fictitious force needed in the rotating reference frame used. In the fixed inertial reference frame, the Coriolis force does not exist and instead we have the Coriolis acceleration.

The distribution of $u_{1}$ along the film thickness corresponding to the peak near $\frac{\omega}{\omega_{0}}=12$ in Fig. 2 is shown in Fig. 4. It is a higher-order TSh mode which also results in a selfequilibrated Coriolis force system. Geometrically, $\partial u_{1} / \partial x_{3}$

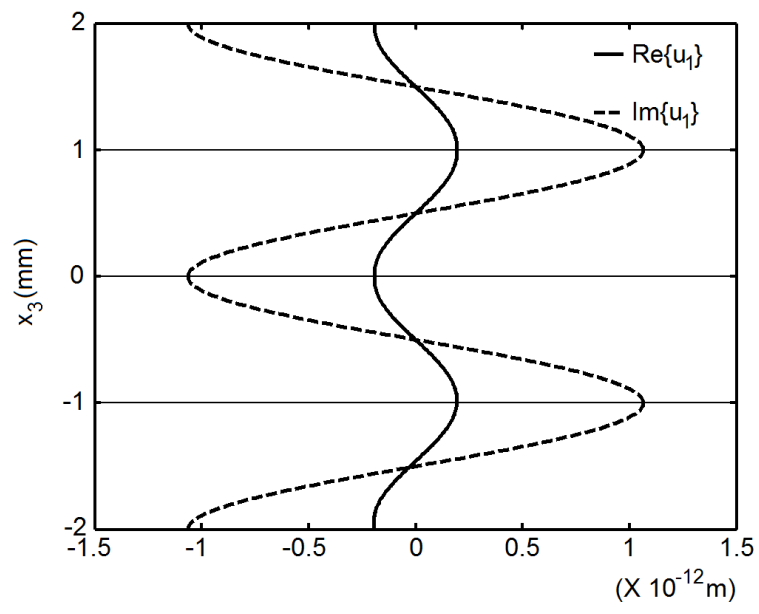

(a)

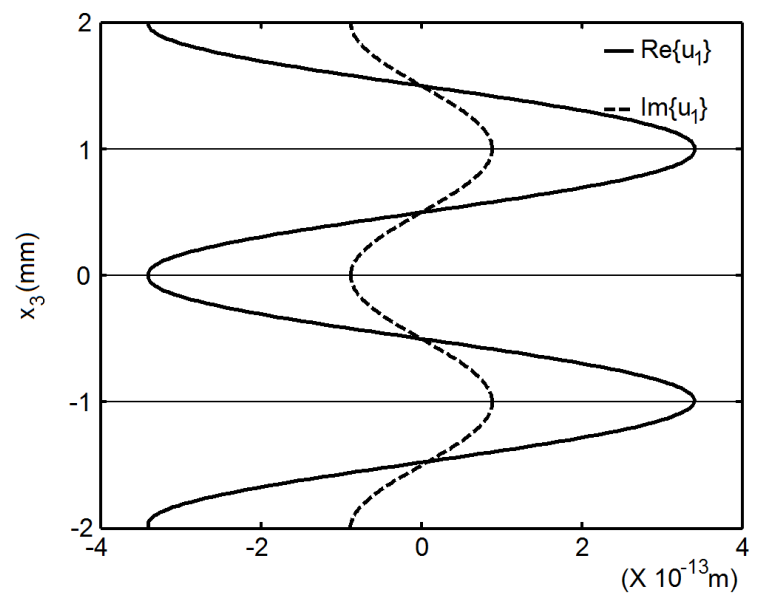

(b)

Figure 3. Distribution of $u_{1}$ along the film thickness $\left(\Omega=0.02 \omega_{0}, Z=\right.$ $\left.0.3 Z_{0}\right)$. (a) Corresponding to the left peak near $\frac{\omega}{\omega_{0}}=4$ in Fig. 2. (b) Corresponding to the right peak near $\frac{\omega}{\omega_{0}}=4$ in Fig. 2.

describes the TSh strain due to $u_{1}\left(x_{3}\right)$. In Fig. 4, the TSh strain changes its sign within each of the four layers. This causes cancellation of the charge (or voltage) produced by the shear strain across the output electrodes through piezoelectric coupling. Hence the output voltage is relatively low near $\frac{\omega}{\omega_{0}}=12$ in Fig. 2.

In Fig. 5, we plot the output voltage of the left resonance near $\frac{\omega}{\omega_{0}}=4$ versus $\Omega$, the angular rate to be measured. The figure shows that a higher output voltage corresponds to a larger $\Omega$ than expected. In particular, when $\Omega$ is relatively small compared to $\omega_{0}$, there is an essentially linear relationship between the output voltage and $\Omega$ which is ideal for a sensor. The plate is very thin with a very high $\omega_{0}$. Therefore, the essentially linear range with $\Omega<<\omega_{0}$ covers a wide range of applications.

Figure 6 shows the effect of $Z$, the impedance of the output circuit, on the output voltage for the left resonance near $\frac{\omega}{\omega_{0}}=$ 4 . For small $Z$, the output circuit is nearly shorted. In this case, although the output voltage is low, the output current must be high which can also be used to measure $\Omega$. As $Z$ increases from zero, there is an essentially linear range. When $Z$ is large, the output circuit is nearly open. In this case the output voltage is large and saturates, and the output current is small. 


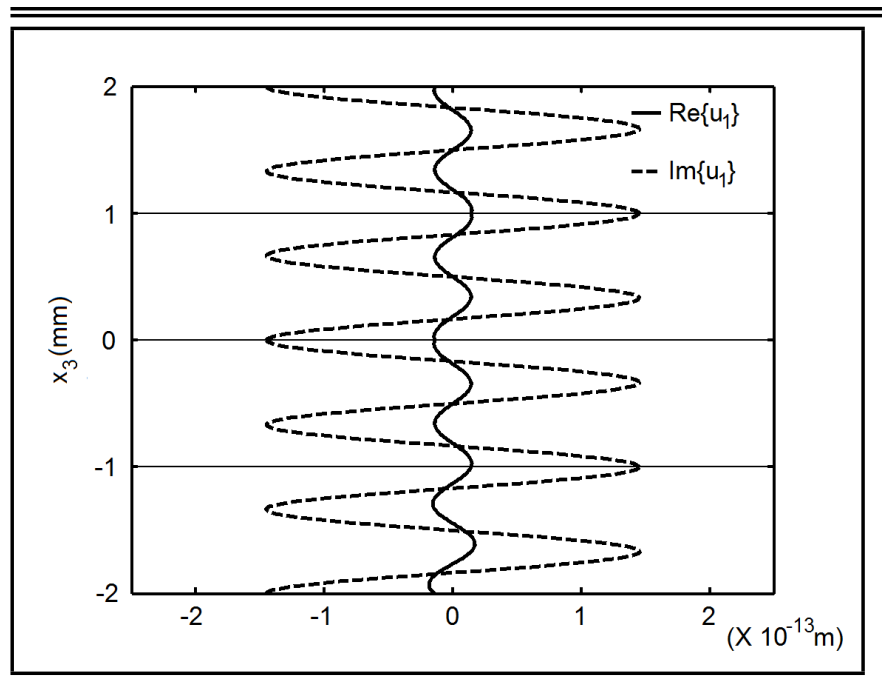

Figure 4. Distribution of $u_{1}$ along the film thickness near $\frac{\omega}{\omega_{0}}=12$ in Fig. 2 $\left(\Omega=0.02 \omega_{0}, Z=0.3 Z_{0}\right)$.

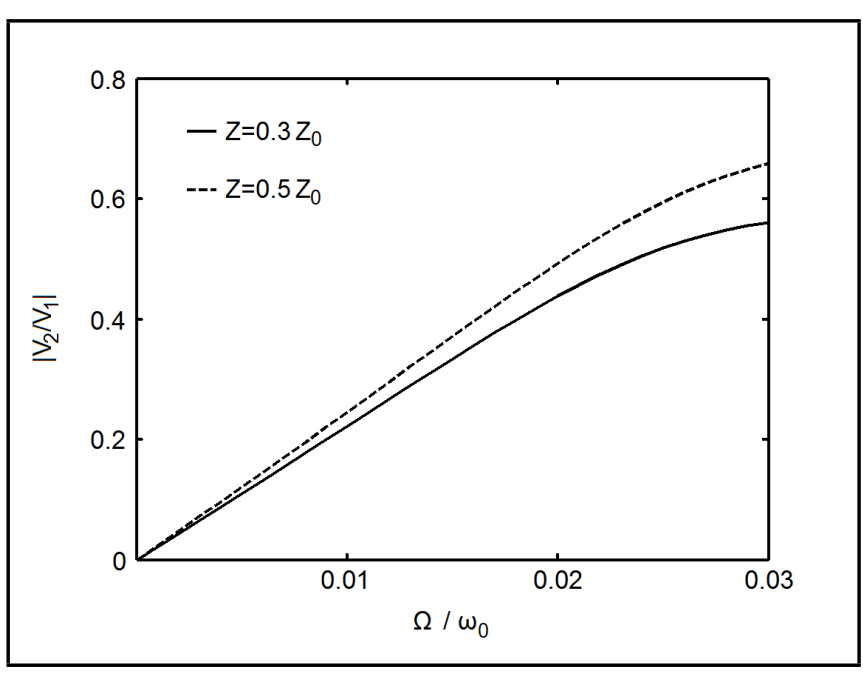

Figure 5. Output voltage versus $\Omega$ for the left peak near $\frac{\omega}{\omega_{0}}=4$ in Fig. 2.

For a complete view, we plot the output voltage versus both the angular rate and the load impedance in Fig. 7. It can be seen that the output voltage is an increasing function of both $\Omega$ and $Z$, and is essentially linear when they are low values.

Figure 8 shows the effects of layer thicknesses on the output voltage near $\frac{\omega}{\omega_{0}}=4$. The solid line is for the case of identical layer thickness. For the dotted line, the total film thickness is kept the same as that of the solid line but the thicknesses of the individual layers were varied slightly. In this case the TSh strain corresponding to the modes in Fig. 3 no longer change its sign exactly at the interfaces among different layers. As a consequence, there is some charge cancellation at the output electrodes, which lowers the peaks. The resonant frequencies are also affected by the layer thicknesses. These frequencies mainly depend on the material density, the relevant shear elastic constant $c_{55}$ and the film total thickness. However, since the material is piezoelectric, the frequencies also depend on the electric field in the film and the related piezoelectric stiffening effect. Varying layer thicknesses changes the electrical state in the layers and thus affect the frequencies.

In Fig. 9, the solid line represents the case of a square film with $2 a=2 b$. For the dotted line, the dimension of the film along $x_{2}$ is increased a little. This affects the electric field and the output voltage through $E_{2}=-V_{2} /(2 b)$. Since the

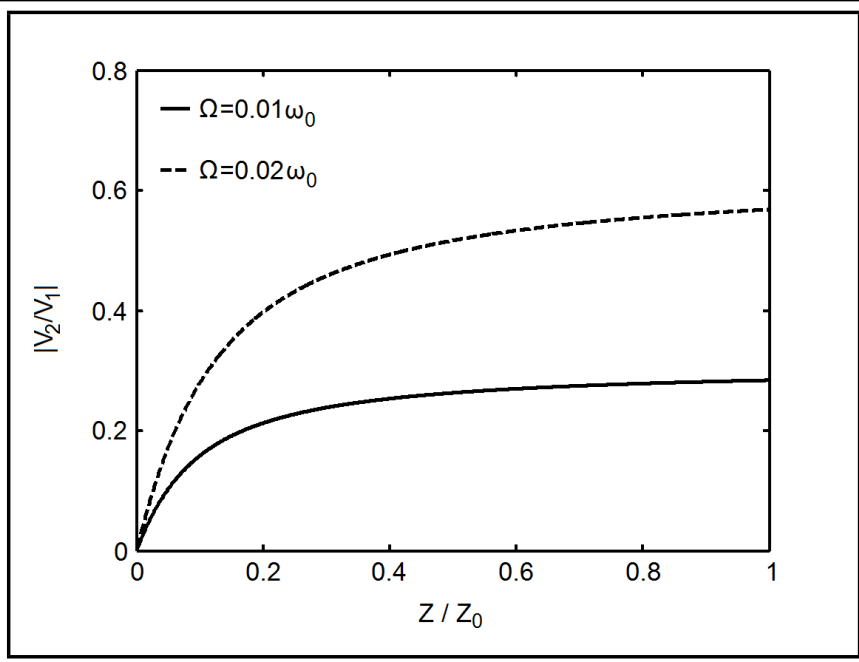

Figure 6. Output voltage versus $Z$ for the left peak near $\frac{\omega}{\omega_{0}}=4$ in Fig. 2.

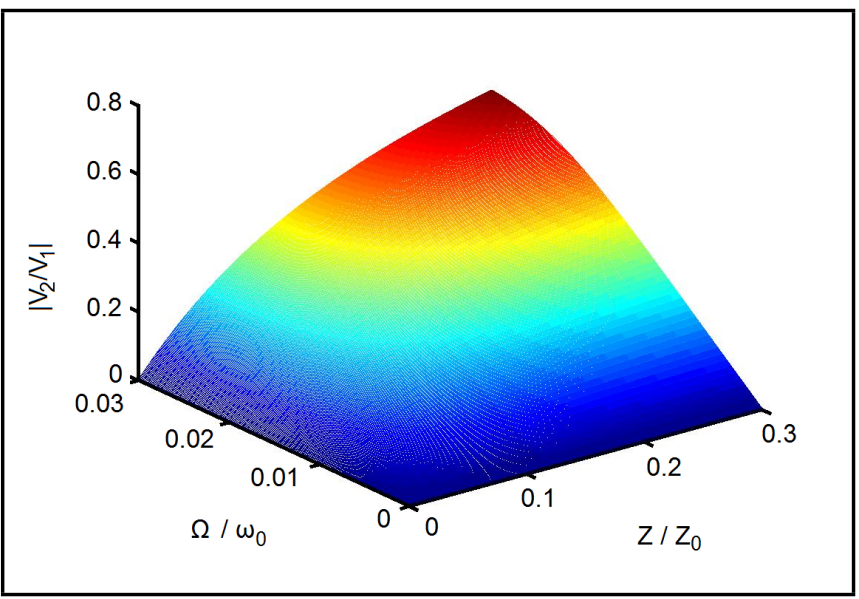

Figure 7. Output voltage versus both $\Omega$ and $Z$ for the left peak near $\frac{\omega}{\omega_{0}}=4$ in Fig. 2.

electrical state in the structure is affected, the frequencies and the output voltage also change accordingly.

\section{CONCLUSIONS}

It is shown that a piezoelectric gyroscope can operate with higher-order overtone TSh modes in a multilayered thin film of AlN or $\mathrm{ZnO}$. The gyroscope has an important advantage, i.e., the Coriolis force due to rotation forms a self-equilibrated system and does not cause reactions at mounting points. Therefore, the device has little energy leakage through mounting and potentially has a high quality factor. The proposed gyroscope has high resonant frequencies and potentially high sensitivity. The output voltage of the gyroscope is sensitive to the structural parameters which can be used to optimize its design. The analysis in the present paper can be generalized to thin films with more than four layers, and similar results can be expected.

\section{ACKNOWLEDGEMENTS}

This work was supported by the National Natural Science Foundation of China (Nos. 11672141 and 11372145), the Y. K. Pao Visiting Professorship at Ningbo University, and the K. C. Wong Magana Fund through Ningbo University. 


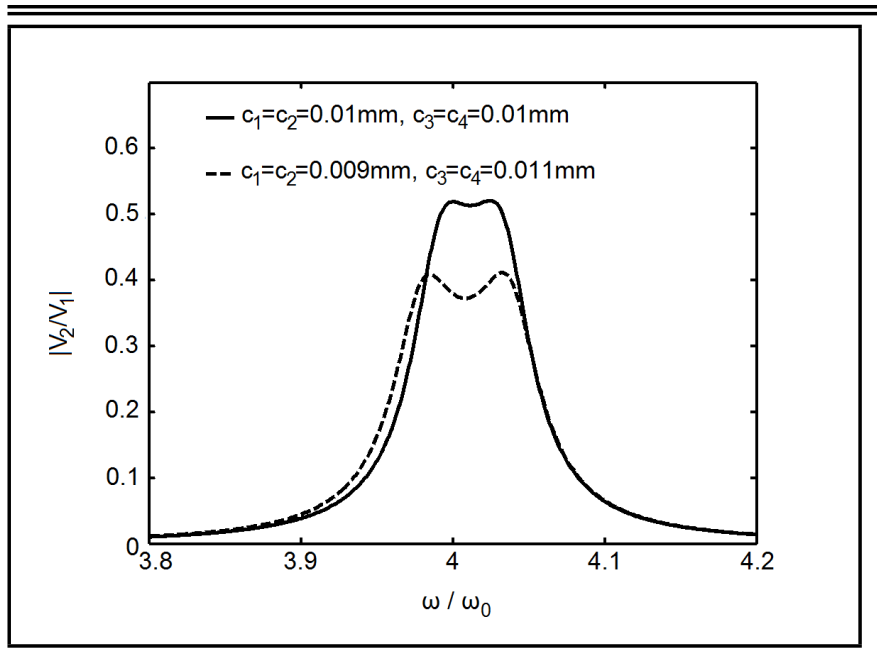

Figure 8. Effect of layer thickness near $\frac{\omega}{\omega_{0}}=4\left(\Omega=0.02 \omega_{0}, Z=0.3 Z_{0}\right)$.

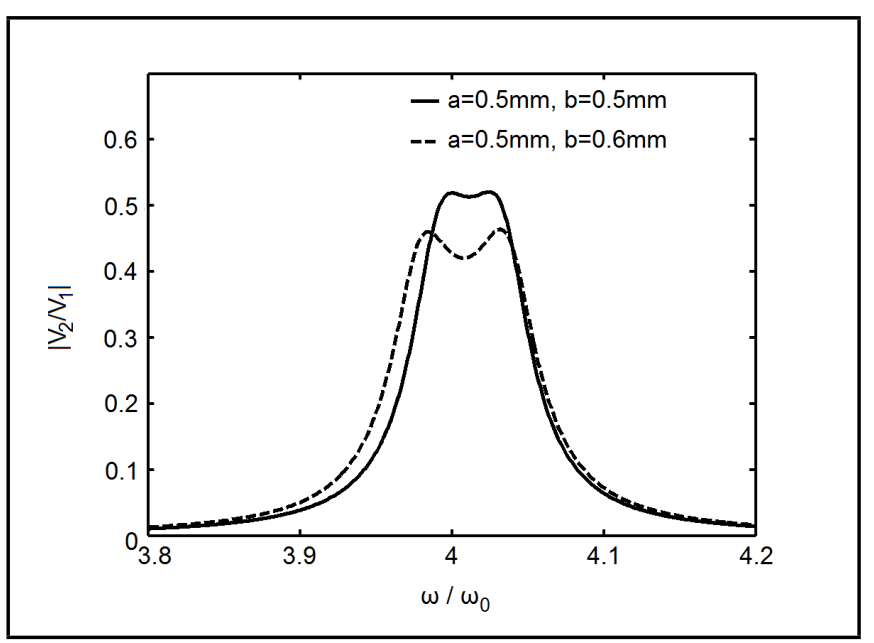

Figure 9. Effects of film size near $\frac{\omega}{\omega_{0}}=4\left(\Omega=0.02 \omega_{0}, Z=0.3 Z_{0}\right)$.

\section{REFERENCES}

1 Gates, W. D. Vibrating angular rate sensor may threaten the gyroscope, Electronics, 41, 130-134, (1968).

2 Yang, J. S. A review of analyses related to vibrations of rotating piezoelectric bodies and gyroscopes, IEEE Transactions On Ultrasonics, Ferroelectrics, And Frequency Control, 52 (5), 698-706, (2005). https://dx.doi.org/10.1109/TUFFC.2005.1503958

3 Yang, J. S. Analysis of ceramic thickness shear piezoelectric gyroscopes, J. Acoust. Soc. Am., 102 (6), 3542-3548, (1997). https://dx.doi.org/10.1121/1.420398

4 Abe, H., Yoshida, T., Ishikawa, T., Miyazaki, N., and Watanabe, H. Trapped energy gyroscopes using thickness shear vibrations in partially polarized piezoelectric ceramic plate, Jpn. J. Appl. Phys., 37 (9S), 5345-5348, (1998). https://dx.doi.org/10.1143/JJAP.37.5345

5 Nakamura, K. and Ohsaki, M. Trapped-energy vibratory gyroscopes using rotated Y-cut LiNbO3, Jpn. J. Appl. Phys., 37 (5S), 2864-2867, (1998). https://dx.doi.org/10.1143/JJAP.37.2864

6 Yang, J. S., Fang, H. Y., and Jiang, Q. Equations for a piezoelectric parallelepiped and applications in a gyroscope, Int. J. of Appl. Electromagnetics and Mechanics, 10 (4), 337350, (1999). https://dx.doi.org/10.3233/JAE-1999-152
7 Watanabe, H., Abe, H., Takahashi, M., and Ishikawa, T. Trapped-energy vibratory gyroscopes using piezoelectric ceramic plate with plano-mesa structure, Jpn. J. Appl. Phys., 42 (5S), 3396-3398, (2002). https://dx.doi.org/10.1143/JJAP.41.3396

8 Cui, J., Du, J. K., Wang, J., and Yang, J. S. A piezoelectric gyroscope based on thicknessshear modes of an AIN bimorph with inclined C axes, Phil. Mag. Lett., 94 (7), 447-454, (2014). https://dx.doi.org/10.1080/09500839.2014.923945

9 Wang, Z., Zhao, M. H., and Yang, J. S. A piezoelectric gyroscope with self-equilibrated Coriolis force based on overtone thickness-shear modes of a lithium niobate plate with an inversion layer, IEEE Sens. J., 15 (3), 1794-1799, (2015). https://dx.doi.org/10.1109/JSEN.2014.2366235

10 Yanagitani, T., Morisato, N., Takayanagi, S., Matsukawa, M., and Watanabe, Y. C-axis zig-zag $\mathrm{ZnO}$ film ultrasonic transducers for designing longitudinal and shear wave resonant frequencies and modes, IEEE Trans. Ultrason., Ferroelect., Freq. Contr., 58 (5), 1062-1068, (2011). https://dx.doi.org/10.1109/TUFFC.2011.1906

11 Wu, X. S., Chen, W. Y., Lu, Y. P., Xiao, Q. J., Ma, G. Y., Zhang, W. P., and Cui, F. Vibration analysis of a piezoelectric micromachined modal gyroscope (PMMG), J. Micromech. Microeng., 19 (12), 125008-125017, (2009). https://dx.doi.org/10.1088/0960-1317/19/12/125008

12 Kotru, S., Highsmith, A., Zhong, J. A., Rincon, V., Jackson, J. E., and Ashley, P. Feasibility study of a micromachined single-axis vibratory gyroscope using piezoelectric PNZT thin films for actuation and sensing, Smart Mater. Struct., 19 (8), 085001, (2010). https://dx.doi.org/10.1088/0964$1726 / 19 / 8 / 085001$

$13 \mathrm{Hu}$, X. J., Wu, X. S., Wang, Z., Chen, W. Y., and Zhang, W. P. Model Design of Piezoelectric Micromachined Modal Gyroscope, Journal of Sensors, 2011, art. 106482, (2011). https://dx.doi.org/10.1155/2011/106482

14 Tabrizian, R., Hodjat-Shamami, M., and Ayazi, F. HighFrequency AlN-on-Silicon Resonant Square Gyroscopes, J. Microelectromech. S., 22 (5), 1007-1009, (2013). https://dx.doi.org/10.1109/JMEMS.2013.2273031

15 Xia, D. Z., Yu, C., and Kong, L. The Development of Micromachined Gyroscope Structure and Circuitry Technology, Sensors, 14 (1), 1394-1473, (2014). https://dx.doi.org/10.3390/s140101394

16 Zhang, H. F. and Bao, Y. Y. Sensitivity analysis of multi-layered $\mathrm{C}$-axis inclined zigzag zinc oxide thinfilm resonators as viscosity sensors, IEEE Trans. Ultrason., Ferroelect., Freq. Contr., 61 (3), 525-534, (2014). https://dx.doi.org/10.1109/TUFFC.2014.2936

17 Tiersten, H. F. Linear Piezoelectric Plate Vibrations: Elements of the Linear Theory of Piezoelectricity and the Vibrations of Piezoelectric Plates, Plenum Press, New York, (1969).

18 Tsubouchi, K., Sugai, K., and Mikoshiba, N. AlN material constants evaluation and SAW properties on AlN/A12O3 and AlN/Si, Proc. IEEE 1981 Int. Ultrasonics Symp., 375-380, (1981). https://dx.doi.org/10.1109/ULTSYM.1981.197646 